\title{
The value in just "doing it": Assessing competence and performance in pediatric urology
}

\author{
Robert Stewart, MD, FRCSC
}

Division of Urology, St. Michael's Hospital, Toronto, ON

See related article on page 317.

Cite as: Can Urol Assoc J 2014;8(9-10):321-2. htrp://dx.doi.org/10 .5489/cuaj.2295

Published online October 13, 2014.

$\mathrm{O}$ bjectives for training in urology are benchmarks set out by the Specialty Committee in Urology of the Royal College. Pediatric Urology represents an area of subspecialization where these objectives are extremely important. This is due, at least in part, to the fact that pediatric urology is a shifting landscape - the procedural platform is constantly changing in light of evolving evidence.

For example, the treatment of vesicoureteral reflux has changed dramatically in the past decade. What was once a bilateral re-implant has become, in many cases, a procedure of subtrigonal injection. Antenatal diagnosis of hydronephrosis has changed the approach to ureteropelvic junction (UPJ) obstruction. In addition, some training programs are limited in their exposure to pediatric urology procedures. The Royal College objectives document is an iterative exercise that is examined by the Specialty Committee at regular intervals to confirm that the objectives for training are both meaningful and attainable.

Gustafson and colleagues provide descriptive evidence from 6 training programs in Canada and examine their exposure to pediatric urology cases that have been designated as levels A, B and C in the objectives document. ${ }^{1}$ The results of this survey are provocative. There is wide variability in the exposure of trainees to the A level cases, but generally it is a fairly robust experience, one that leads to confidence that the residents possess competence in these procedures. Beyond that, there is a floor effect across programs, with many trainees never being exposed to $B$ level cases, and almost none had exposure to $C$ level cases. This paper represents an incomplete sampling of training programs in Canada (6 out of 12) and the degree of involvement in these cases by resident trainees was not specified. It is unclear whether the observations presented here are generalizable to the entire Canadian urology training experience. Guidelines for survey methodology have been previously published ${ }^{2}$ and highlight the potential pitfalls of this design. However, this study does raise some important issues for discussion. Do we need to frame-shift this categorization to reflect the reality of experience? Clearly, competence is achieved in many of the level A procedures. What is the relevance of levels B and $C$ ? Can we provide more exposure for our trainees to satisfy the criteria for a level B distinction?

As we move towards competency-based training, there is an opportunity to reflect on the important distinction between competency and competence. The former may reflect a technical skill devoid of clinical context, and not requiring judgment or any of the cognitive components of surgery (i.e., laparoscopic peg transfer). Competence reflects an element of mastery, implying that the cognitive and technical components have been synthesized and that the individual possesses a global facility in a particular procedure. Achieving competence does not necessarily reflect on the learning process of the competency in question. Going forward into this new paradigm, we must be careful to not reduce competence to competencies.

In Urology, fellowship training has become almost a standard. In pediatric urology training centres, the fellows have requirements for certification, benchmarks for "index cases" and this may affect the exposure or at least the degree of involvement that a resident may have with a fellow in a particular case. As the index cases change with time, so may the acuity of a fellow's need to reach these minimum standards. What is learned by a resident in a procedure if not "doing it?" There is value in observational learning, but it is difficult to quantify, and can be highly variable. ${ }^{3}$ As we progress through this evolution towards competency-based training, it is hoped that we can identify better benchmarks for performance in all areas of our specialty. Insights into 
the learning process surrounding the acquisition of competence will be welcomed. Are we teaching to the pediatric component of the Royal College exam, or are we adequately preparing our trainees to do some pediatric urology in their practice? Pediatric urology will remain a difficult subspecialty area to assess, but rigorous reconciliation of what our objectives are and the experience of our trainees is an important aspect of sustained reflection within our educational model. It is critical that we remain open to adapting our educational strategies to achieve these objectives. It is also important that the Royal College Specialty Committee remain vigilant and continue to redefine the objectives in this changing field.

Competing interests: Dr. Stewart declares no competing financial or personal interests.

\section{References}

1. Gustafson P, MacNeily A. Pediatric procedures in urology resident training: An analysis of the experience of Canadian urology residents. Can Urol Assoc J 2014;8:317-20. http://dx.doi.org/10.5489/cuai.2004

2. Burns KEA, Duffett $M$, Kho ME, et al. A guide for the design and conduct of self-administered surveys of clinicians. CMAJ 2008;179:245-52. http://dx.doi.org/10.1503/cmaj.080372

3. Bandura A. Social learning theory. New York: General Learning Press; 1971.

Correspondence: Dr. Robert Stewart, St. Michael's Hospital, $9111-61$ Queen St. East, Toronto, ON M5C 2T2; stewartr@smh.ca 